\title{
Towards the Large-Eddy Simulation of a full engine: Integration of a 360 azimuthal degrees fan, compressor and combustion chamber. Part II: Comparison against stand-alone simulations
}

\section{Original article}

Article history:

Submission date: 16 October 2020

Final revision date: 18 December 2020

Acceptance date: 6 February 2021

Publication date: 25 May 2021

Check for updates

*Correspondence:

JD: dombard@cerfacs.fr

\section{Peer review:}

Single blind

\section{Copyright:}

(c) 2021 Pérez Arroyo et al. @ This is an open access article distributed under the Creative Commons Attribution Non Commercial No Derivatives License (CC BY-NC-ND 4.0). Unrestricted use, distribution, and reproduction of the original work are permitted for noncommercial purposes only, provided it is properly cited and its authors credited. No derivative of this work may be distributed.

\section{Keywords:}

interaction; LES; compressor; combustion chamber; fan; gas-turbine engine

\section{Citation:}

Pérez Arroyo C., Dombard J., Duchaine F., Gicquel L., Martin B., Odier N., and Staffelbach G. (2021). Towards the LargeEddy Simulation of a full engine: Integration of a 360 azimuthal degrees fan,

compressor and combustion chamber. Part

II: Comparison against stand-alone simulations. Journal of the Global Power and Propulsion Society. Special Issue: DataDriven Modelling and High-Fidelity Simulations: 1-16. https://doi.org/10.33737/jgpps/133116
Carlos Pérez Arroyo ${ }^{1}$, Jérôme Dombard ${ }^{1, *}$, Florent Duchaine $^{1}$, Laurent Gicquel ${ }^{1}$, Benjamin Martin ${ }^{1}$, Nicolas Odier ${ }^{1}$, Gabriel Staffelbach ${ }^{1}$

${ }^{1}$ CERFACS, 42 Avenue Gaspard Coriolis, 31100 Toulouse, France

\begin{abstract}
Unsteady simulations of various components of a gas-turbine engine are often carried out independently and only share averaged quantities at the component interfaces. In order to study the impact and interactions between components, this work compares results from sectoral stand-alone simulations of a fan, compressor and annular combustion chamber of the DGEN-380 demonstrator engine at take-off conditions against an integrated 360 azimuthal degrees large-eddy simulation with over 2.1 billion cells of all previously listed components. Note that, at take-off conditions the compressor works at transonic conditions and generates an upstream-propagating shock that interacts with the fan modifying the shape of its wake with respect to the stand-alone simulation. Furthermore, the shock is seen as a tone in the pressure spectra at half the impeller blade passing frequency in the forward region of the engine. In the aft region, time-averaged fields are overall similar between stand-alone and integrated simulations but show a deviation in the azimuthal position of the hot-spot at the exit of the combustion chamber due to the addition of the diffuser. Pressure fluctuations generated in the compressor are captured in the combustion chamber as tones in the temperature and pressure spectra at the impeller bladepassing frequency and harmonics as well as an increase in the rootmean-square pressure.
\end{abstract}

\section{Introduction}

Increasing reliability and efficiency of gas-turbine engines, while reducing pollutant and noise emissions is one of the main goals of industry. Currently, engines are designed by several departments that focus on different engine components (compressor, combustion chamber, turbine and others). While the individual components may behave within design margins, the assembled engine could exhibit overall poorer performance due to integration effects, i.e. interactions between components that were unknown or not considered in the design phase of each individual component. Here, the integration effects are investigated with a massive 2,100 million cells 360 azimuthal degrees reactive Large-Eddy Simulation (LES) of a turbo-fan engine composed of the fan, compressor and combustion chamber. Performing a single LES, although costlier, 
simplifies the transfer of unsteady phenomena such as turbulence and pressure fluctuations between components since there are no boundaries to define at interfaces.

This work is the continuation of the companion paper (Pérez Arroyo et al., 2021) where the methodology and initialisation to perform such a simulation are explained in detail and are briefly recalled in the following. In order to reduce the convergence time (computational cost), the initial solution of the integrated simulation (referred hereafter as FULLEST) is constructed from stand-alone sectoral LES of the fan, compressor and combustion chamber. This generates as well a three-dimensional data-base of each component used for comparison. The generation of the unstructured mesh of over 2,100 million cells for FULLEST consists of two operations to yield the best trade-off to solve potential machine dependent memory limitations. The domains of the stand-alone simulations that are geometrically isolated such as de fan, the static sub-domain of the outlet-guide vanes (OGVs) and the impeller are repeated azimuthally using the in-house package for manipulating unstructured computational grids HIP (Müller, 1999). The second procedure is applied to the diffusers and the combustion chamber that do not share the azimuthal periodicity. In this case, a coarse mesh is generated first for the full azimuthal extent (360 degrees) from the exit of the impeller to the exit of the combustion chamber. Then, the mesh is refined using the library MMG (Dapogny et al., 2014; Daviller et al., 2017) where a metric is constructed using the meshes from the stand-alone sectoral simulations as the target mesh. FULLEST computation converges to an operating point with less than $0.5 \%$ difference in in mass-flow, total temperature and total pressure compared to the stand-alone simulations with an overall $1 \%$ error if compared to the designed thermodynamic cycle. Last, the flow topology of the impeller is introduced. At take-off conditions, the compressor works at transonic conditions and generates a shock anchored at the leading edge of the impeller. Since, it rotates with it, this discontinuity is seen as a propagation in the absolute frame.

The impact of this shock on the fan stage is studied in more details in this work which is structured as follows. First, the configuration is briefly introduced. Then results of the integrated simulation are analysed and compared against the stand-alone LES predictions of each component for the fan, compressor and the combustion chamber. Finally, main conclusions are listed.

\section{Configuration}

The DGEN-380 demonstrator engine is studied here numerically at take-off conditions in a single reactive wallmodelled LES performed with the explicit unstructured and massively parallel compressible flow solver AVBP developed by CERFACS (Schönfeld and Rudgyard, 1999). The domain contains the full 360 azimuthal degrees LES of: the fan rotating at 13,053 rpm with 14 blades; 42 outlet-guide vanes; the radial compressor composed of 11 main blades and 11 splitter blades rotating at 51,390 rpm (in the opposite direction of the fan), as well as a radial and an axial diffusers composed of 22 and 55 vanes, respectively; and the annular combustion chamber with primary and dilution holes as well as 13 double contra-rotating swirlers. Results are compared against stand-alone simulations of each component that are performed on an azimuthal sector of 360/14 degrees for the fan and OGVs (noted as FAN + OGV), 360/11 degrees for the compressor including the radial and axial diffusers (noted as CoHP) and 360/13 degrees for the combustion chamber (noted as CC). The integrated domain FULLEST shown in Figure 1 is constructed by azimuthal repetition of the sectoral domains to have a fair

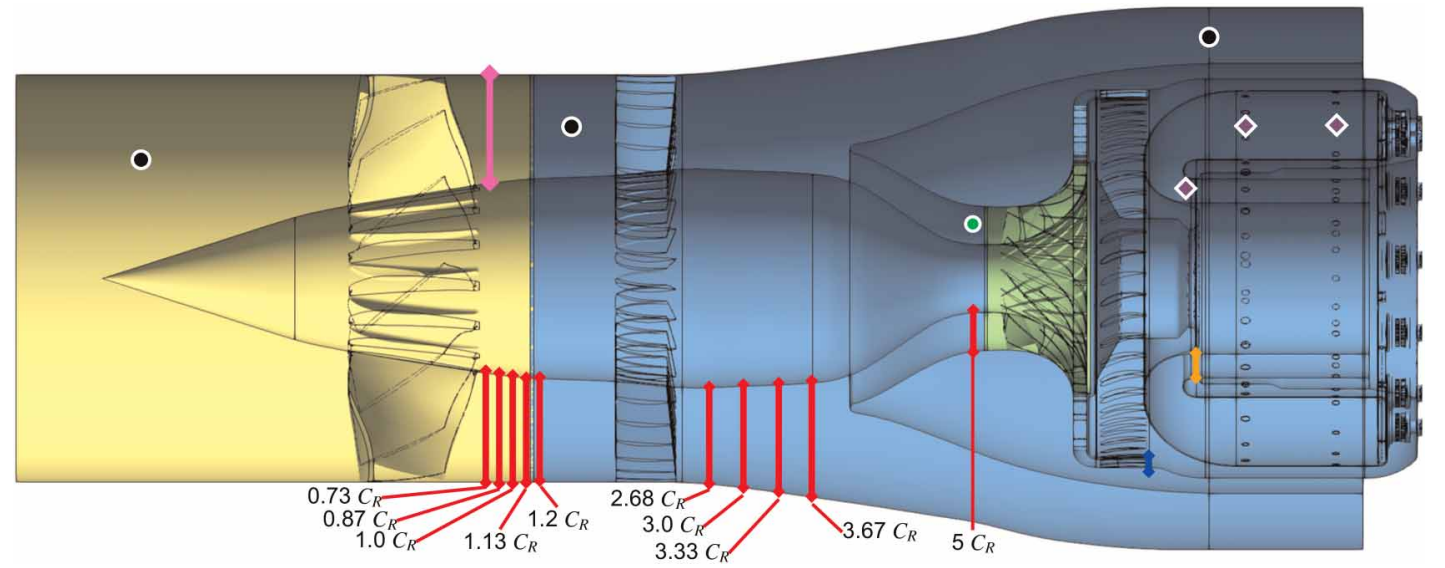

Figure 1. View of the $360^{\circ}$ FULLEST domain. Blue depicts the static sub-domains, and green and yellow the rotating sub-domains depending on its rotational speed. Lines and markers in Figure 1 are used as a reference throughout this work. 
comparison between simulations. Nonetheless, besides their azimuthal extent, the domains still present some differences which are listed in the following. The outlet of the primary duct in FAN + OGV and outlet in CoHP are extended axially to ease the evacuation of turbulence. Similarly, the inlet of CoHP and CC are also axially extended. The diffuser vanes were not considered in CC because its azimuthal periodicity is different than the one for the chamber. More details about the domains, operating point, numerics and mesh specifications can be found in the companion paper (Pérez Arroyo et al., 2021).

\section{Results}

In this section, results from FULLEST are compared against the stand-alone simulations to analyse the impact of the integration on the aerodynamics and acoustics. Time-averaged fields and spectra are presented for all the components starting with the fan, following with the compressor and finalising with the combustion chamber. Three-dimensional information (e.g. axial, meridional or radial cuts) extracted from the integrated simulation are azimuthally averaged onto a single sector (which angles depend on the component of study) in order to increase its statistical convergence. This is performed by splitting the solution for all azimuthal sectors and interpolating it on the mesh of one sector which angles coincide with the stand-alone sectoral simulation. Other results such as the Power Spectral Density (PSD) of local temporal signals are also azimuthally averaged using one signal per sector which has a similar effect as the classical windowing performed in signal analysis.

\section{Comparison of the fan and bypass duct predictions}

As stated in Pérez Arroyo et al. (2021), at take-off conditions, the pressure fluctuations in the bypass duct and the fan are dominated by the shock generated at the transonic impeller. This effect is clearly visible on the static pressure root-mean-square $\left(P_{\text {RMS }}\right)$ as illustrated in Figure 2 for FULLEST and FAN + OGV on a meridional cut and an iso-surface at $50 \%$ of the normalised hub-to-shroud distance (denoted hereafter $h / H$ ). In the stand-alone simulation, pressure fluctuations are mainly generated in three regions: the tip of the fan blades as a result of the tip-leakage vortices (TLV) (Inoue and Kuroumaru, 1989), the wake of the fan and the wake of the OGVs. In FULLEST, $P_{\text {RMS }}$ is simply higher in the whole domain where the TVL is still present, albeit showing on a smaller span region. Additionally, in the primary flow duct, $P_{\mathrm{RMS}}$ contours are saturated because the pressure jump across the shock is greater. Then, they decay towards the OGVs and reach the fan. Pressure RMS patterns are even discernible at the inlet of the fan sub-domain.

Indeed, a tone emerges at half the relative impeller Blade-Passing Frequency (BPF) as illustrated in Figure 3a when the pressure spectra is analysed on numerical probes located before the fan. Here the impeller BPF is considered as relative because the probe is located on the rotating sub-domain of the fan and it rotates with this domain. The relative impeller BPF is computed by adding both rotating speeds of the fan and the impeller (or subtracting them if they were turning in the same direction) before multiplying by the number of blades. In this work, the impeller BPF is computed with all 22 blades of the impeller and thus, the shock which is generated at the trailing edge of the 11 main blades is perceived at half this BPF (relative or not). This peak in the spectrum, hereafter referred to as $C_{s}$, is seen through this whole region in FULLEST including the secondary flow duct as
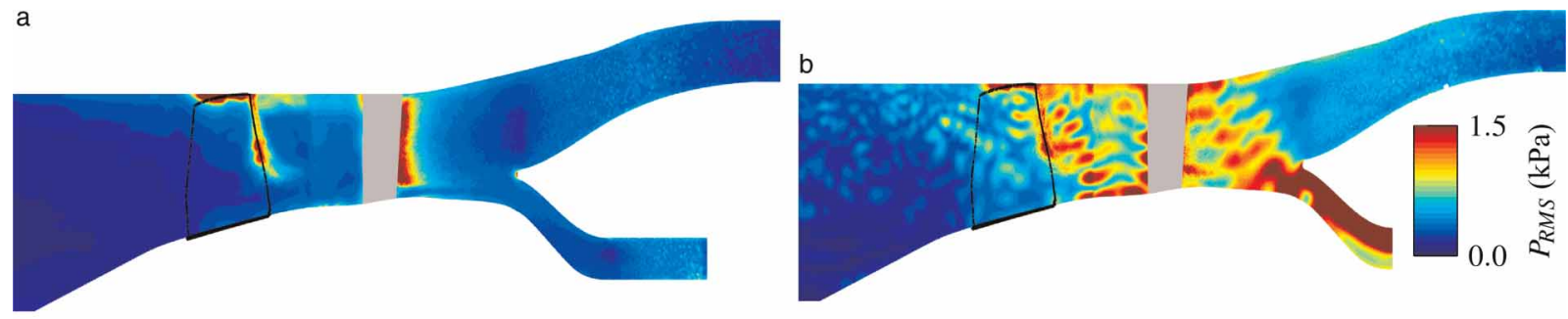

C

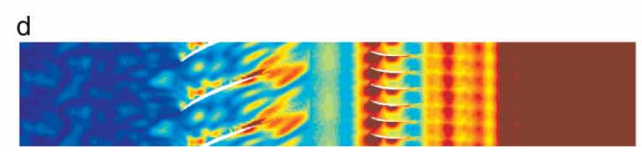

Figure 2. $P_{\mathrm{RMS}}$ contours computed on the respective frame of reference of each sub-domain for (left) FAN + OGV and (right) FULLEST. (a) FAN + OGV: Meridional plane. (b) FULLEST: Meridional plane. (c) FAN + OGV: Iso-surface at $50 \% h / H$. (d) FULLEST: Iso-surface at $50 \% h / H$. 

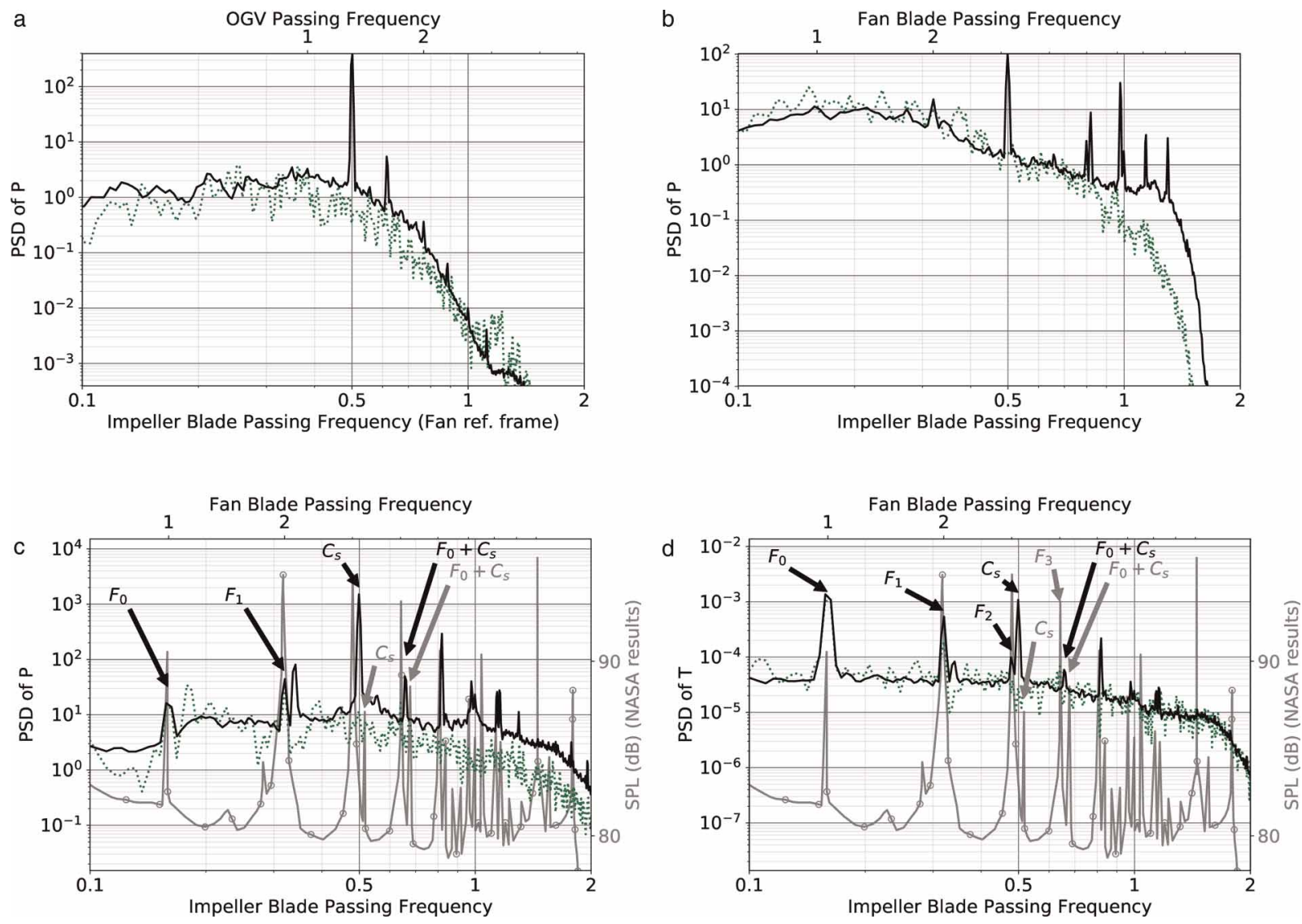

Figure 3. Power spectral density (PSD) at different locations noted in black in Figure 1. (solid black) FULLEST, (dotted green) FAN + OGV, (solid gray) NASA SPL. (a) Inlet of the fan. (b) Secondary flow duct. (c) Position between the fan and the OGVs. (d) Position between the fan and the OGVs.

shown in Figure 3b, even though no pattern is discernible in Figure 2b. Naturally, FAN + OGV spectra do not show $C_{s}$. The flow perceives the wake of the fan, its interaction with the OGVs and the pressure fluctuations from the impeller in FULLEST, between the fan and the OGVs. The resulting spectra at this location are illustrated in Figure $3 \mathrm{c}$ and $3 \mathrm{~d}$ for static pressure $P$ and static temperature $T$, respectively. As a reference, these results are compared against the experimental Sound Pressure Level (SPL) recently measured by Brown and Sutliff (2018) from NASA at similar operating conditions ( $92 \%$ and $95 \%$ of the fan and impeller rotating speeds used in this work, respectively), and for which the frequency is normalised with its respective fan BPF. The fan $\mathrm{BPF}$ tone $F_{0}$ for pressure (Figure $3 \mathrm{c}$ ) and only its first harmonic $F_{1}$ are visible in the LES predictions. The missing harmonics in FULLEST are present, but are hidden below the background noise due to a poor signal-to-noise ratio and would eventually emerge with a longer simulation time. In fact, the third harmonic $F_{2}$ is discernible when the temperature spectrum is analysed (Figure 3d). Above the $C_{s}$ frequency, the combination between $F_{0}$ and $C_{s}$ leads to several interaction tones $C_{s}+F_{n}$, visible in FULLEST and in the experimental results. Note that experimental interaction tones and $C_{s}$ are shifted in frequency if compared to FULLEST because of the different fan to impeller rotational speed ratios.

As a first attempt to unravel the possible impact of such intense pressure fluctuations on the flow topology, instantaneous contours of Q-criterion coloured by $u_{x}$ are shown in Figure 4. In this work, law-of-the-wall boundary conditions are used to reduce the cost of the simulations and thus, boundary layer physics must be carefully analysed (Papadogiannis et al., 2014). Despite the above-mentioned difference in pressure fluctuations, both simulations appear to generate similar vortical structures in this region. Nonetheless, the development of the vortical structures on the fan suction side is more sudden in FULLEST with structures generated directly towards the leading edge as opposed to FAN + OGV where they start to develop in the leading edge.

In order to further study the impact of $C_{s}$ on the boundary layer of the fan, the Discrete Fourier Transform (DFT) is performed on 1,000 instantaneous axial cuts on the wake of the fan (in the rotating frame of reference) equispaced by $10 \mu \mathrm{s}$. The DFT is performed for FULLEST on $u_{x}$ as well as on $P$ and is compared against the RMS values of both FAN + OGV and FULLEST. The spectra for the average over the cut is shown in Figure $5 f$. 

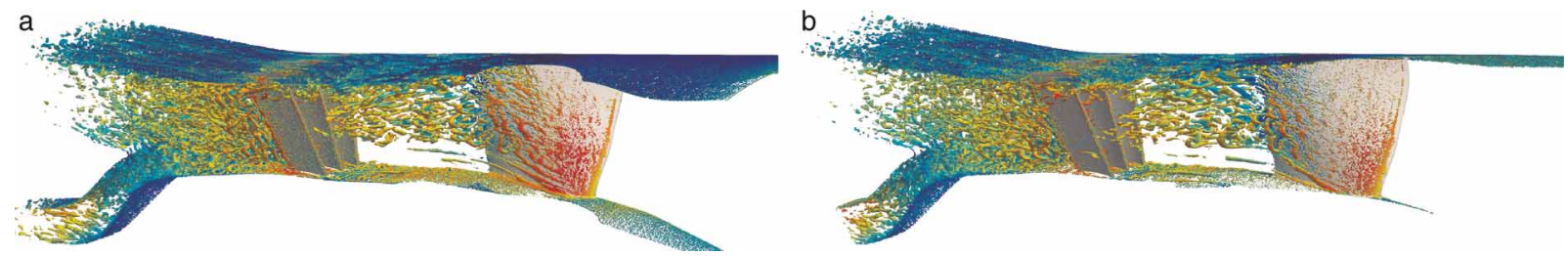

Figure 4. Instantaneous $Q$-criterion contours coloured by $u_{x}$ on the fan suction side and OGVs pressure side. (a) FAN + OGV. (b) FULLEST.

Both $u_{x}$ and $P$ measure a peak at the $C_{s}$ frequency (expressed here in the relative frame of reference of the fan). In addition, in the rotating frame of reference, the OGVs seem to rotate in the opposite direction of the fan. The effect of the OGVs is demonstrated by a secondary smaller peak at the OGV BPF, computed using the rotational speed of the fan and the number of vanes of the OGV. The wake is turbulent with a sinusoidal shape and several thicker regions that correspond to different vortices developed on the fan for both LES. However, there are slight differences on the positioning of the regions of high RMS due to the different development of the boundary layer. The actual profiles of the wake are further analysed in the following. The RMS is by construction, the integral area of the DFT over all frequencies and represents the total contribution of all fluctuations, regardless of its frequency. The mode corresponding to the $C_{s}$ frequency can therefore be extracted with DFT as illustrated in Figure 5e. The intense regions in the PSD for $u_{x}$ demonstrate that the boundary layer is excited at this frequency generating and shedding vortical structures that create a distinct pattern not visible at other frequencies. As expected, $P_{\text {RMS }}$ for FAN + OGV shown in Figure $5 \mathrm{~g}$ only presents the fluctuations generated by the TLV and the wake. In FULLEST, the interaction between the wake and $C_{s}$ features azimuthal and radial patterns in the DFT mode (Figure 5i) as well as for $P_{\mathrm{RMS}}$ map (Figure 5h). The similarity of both fields highlights that the major contribution for the pressure fluctuations is correlated to $C_{s}$.
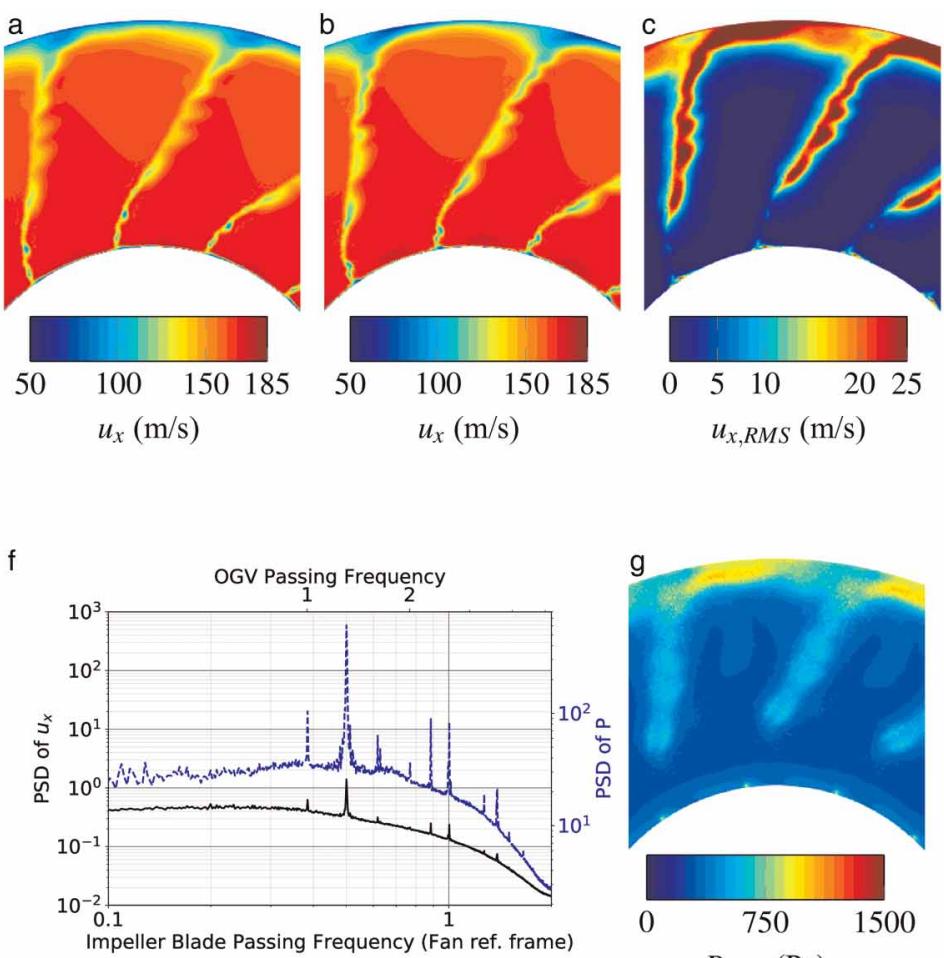

Integral of DFT at all frequencies. (black) $u_{x}$, (blue) $P$.

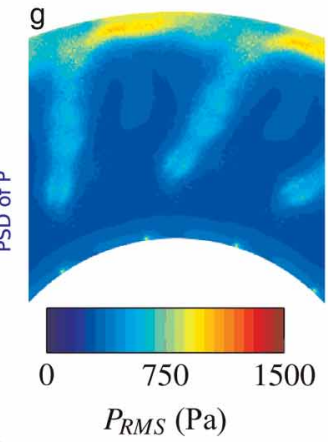

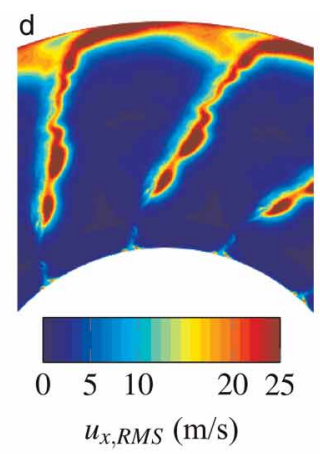
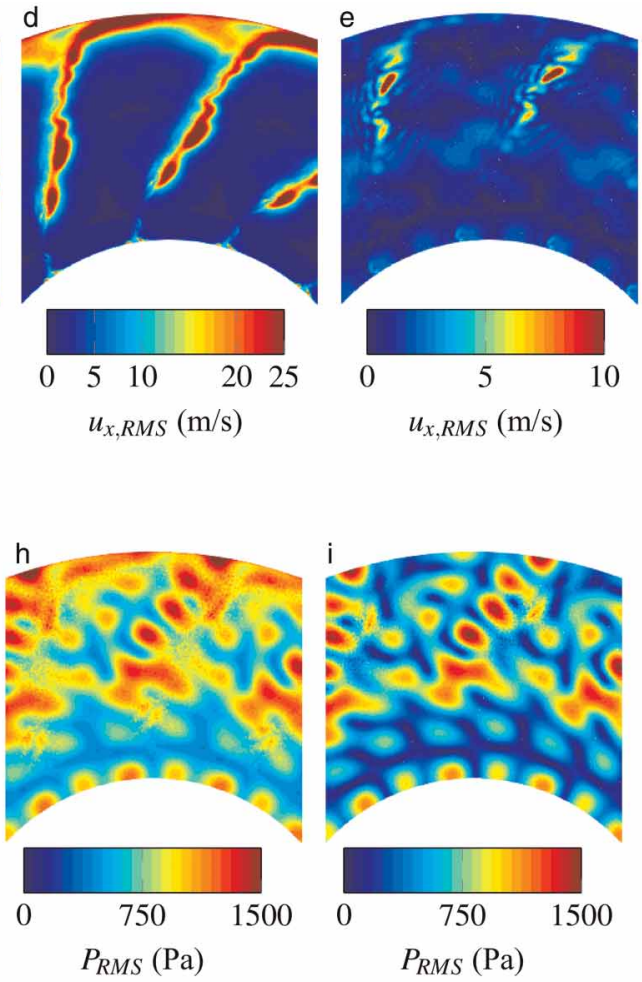

Figure 5. PSD and Contours at the axial position noted in magenta in Figure 1. (a) FAN + OGV. (b) FULLEST. (c) FAN + OGV. (d) FULLEST. (e) FULLEST (DFT). (f) Integral of DFT at all frequencies. (black) $u_{x}$, (blue) $P$. (g) FAN + OGV. (h) FULLEST. (i) FULLEST (DFT). 
The impact of $C_{s}$ on the mean flow is quantified in the following by analysing azimuthal and radial profiles at different axial positions. The azimuthal profiles for the time-averaged and RMS axial velocity extracted at 50\% $h / H$ in the rotating domain (Figure $6 a$ and $6 c$ ) show a slightly stronger velocity deficit after the fan for FULLEST. In these plots the actual $h / H$ is considered (i.e. computed considering the radii at each axial location). Because they are computed in the relative reference frame of the fan, the time-averaged values correspond to the deterministic fluctuation related to the blade-passing motion and the RMS can be considered as the stochastic fluctuations (Adamczyk, 1984; Sharma et al., 1985). The wakes of both LES follow similar profiles and reach turbulence levels of almost $25 \%$ near the trailing edge which decays to $12.5 \%$ after 0.3 fan blade chords noted as $C_{R}$. The wake of the OGVs is depicted in Figure 6b and 6d. The velocity deficit is higher as well for FULLEST at this location. Both wakes decay at similar rates although the wake for FAN + OGV broadens faster which could be an effect of its limited angular sector.

Last, the azimuthally averaged radial profiles are analysed on the static sub-domain and shown in Figure 7. The time-averaged wake of the fan (Figure 7a) presents similar results for both simulations which demonstrates that even if the wakes have slightly different velocity deficits, their azimuthally averaged value remains the same. This also applies to the development of the TLV near the shroud which is not impacted by $C_{s}$. In terms of RMS (Figure $7 \mathrm{~d}$ ), since they are measured in the static domain, they now represent the fluctuation related to the bladepassing motion. Both profiles presents overall similar levels of RMS over the whole height. Nonetheless, the peaks near the hub generated by the horse-shoe vortex appear closer to the hub for FULLEST as it was illustrated in Figure 4 by the Q-criterion. The radial profiles after the OGV are depicted in Figure 7b and 7e. The averaged profiles are once again similar between FAN + OGV and FULLEST despite the more broaden and decayed velocity deficits on FAN + OGV. The RMS profiles present differences related to the interaction between the fan and the OGVs and ultimately, the development of the boundary layer and corner vortices on the OGVs. In particular, RMS values are higher over $20 \% \mathrm{~h} / \mathrm{H}$ for FAN + OGV closer to the OGVs but reach similar values at farther locations due to mixing. Below 20\% h/H, FAN + OGV profiles remain below FULLEST profiles due to the modification of the horse-shoe vortex generated at the fan, that now impacts at a higher height. In the primary flow and close to the impeller, the mean profiles between FAN + OGV and FULLEST are similar over all the extent of the duct as depicted in Figure 7c. The profiles show a higher velocity near the shroud and a lower velocity near the hub because the flow accelerates on the upper corner (shroud of the primary duct) while decelerates on the lower corner (hub of the primary duct). This is not the case for the profile obtained for CoHP
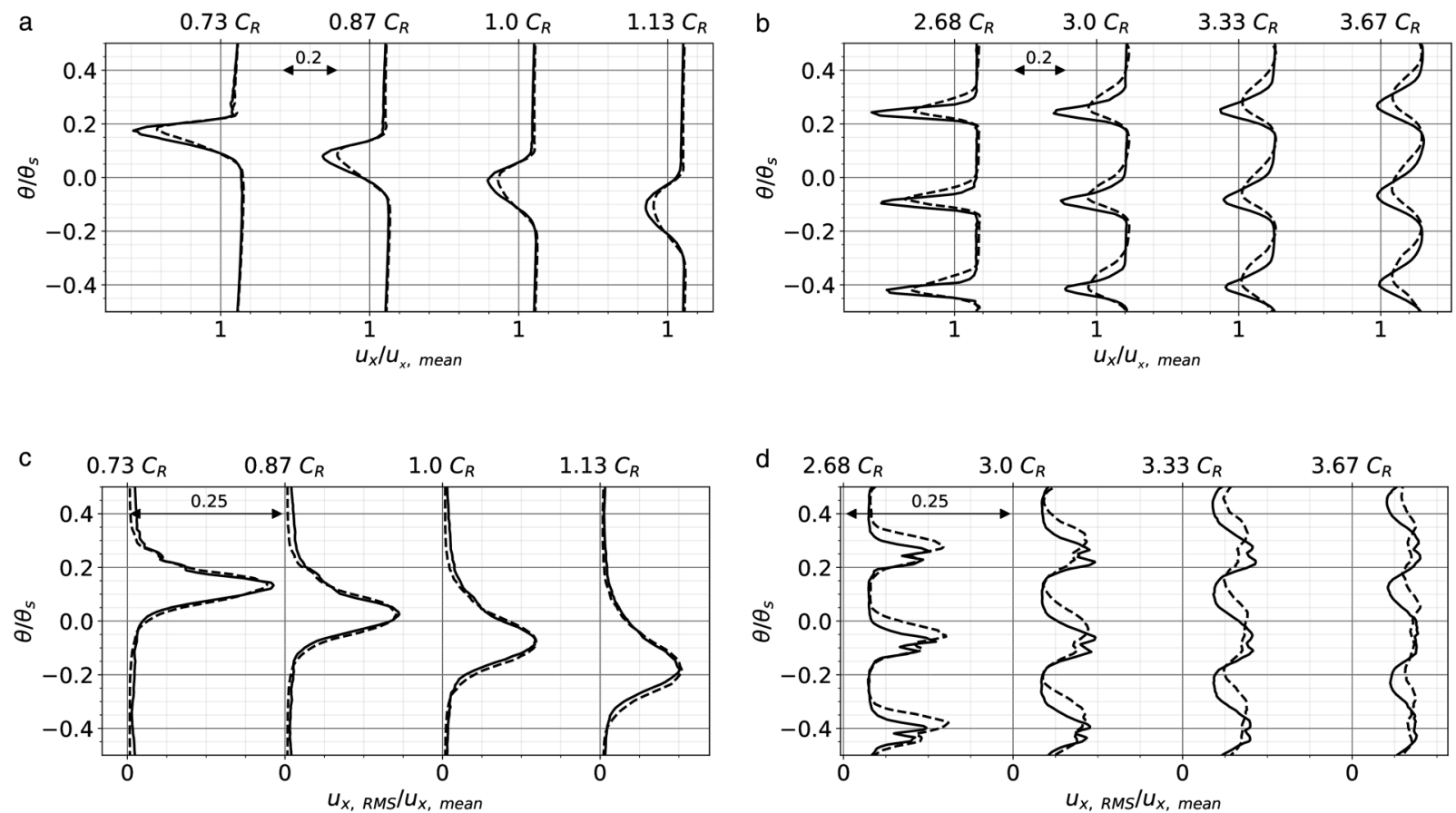

Figure 6. Azimuthal profiles of $u_{x}$ and $u_{x, \text { RMS }}$ on the wake of the fan and OGVs at $50 \% h / H$ and at different axial positions noted in red in Figure 1. Values are normalised by the average axial velocity $\left(u_{x, \text { mean }}\right)$ at each axial location. $\theta_{s}=360 / 14^{\circ} . C_{R}$ is the fan blade chord length at $50 \% \mathrm{~h} / \mathrm{H}$. (solid black) FULLEST; (dashed black) FAN + OGV. (a) Wake of the fan. (b) Wake of the OGVs. (c) Wake of the fan. (d) Wake of the OGVs. 


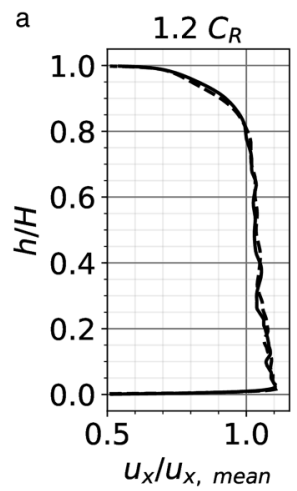

d

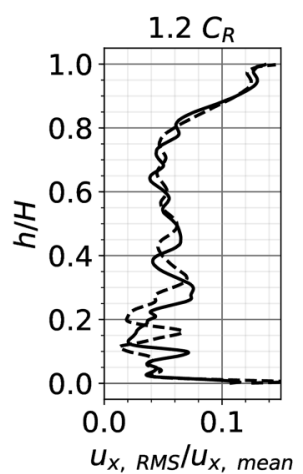

b

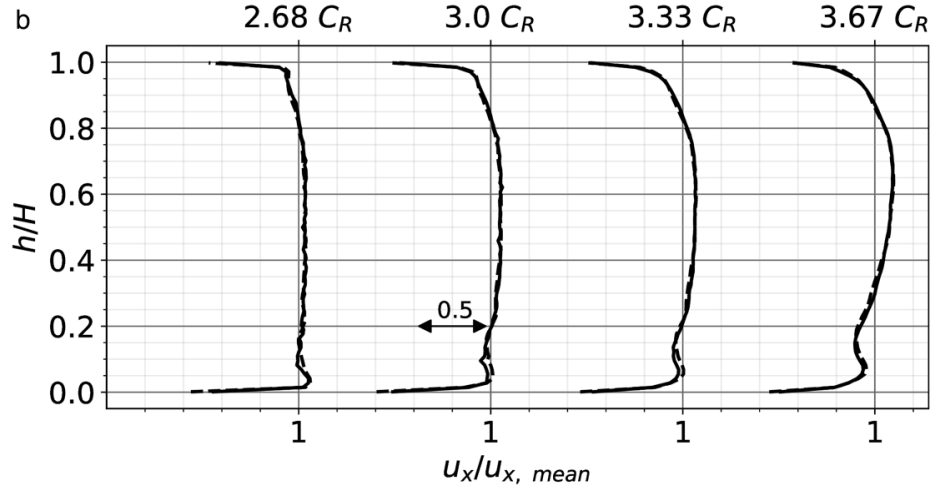

e $\quad 2.68 C_{R}$

$3.0 C_{R}$

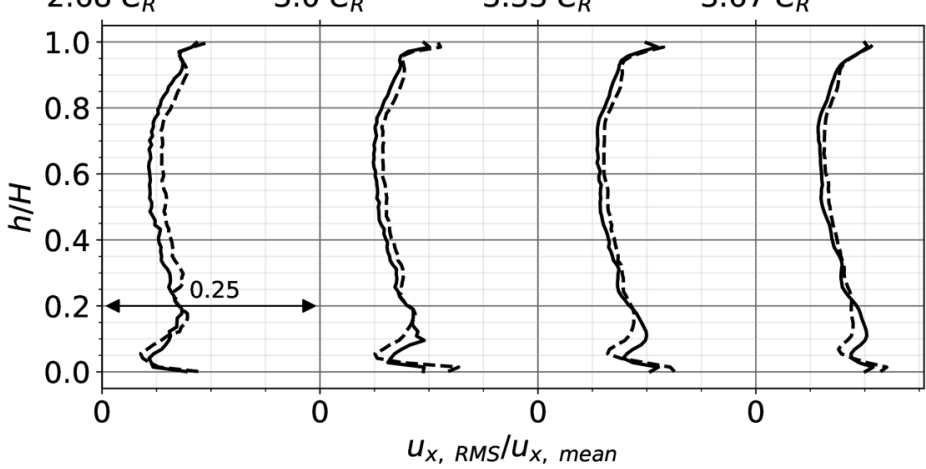

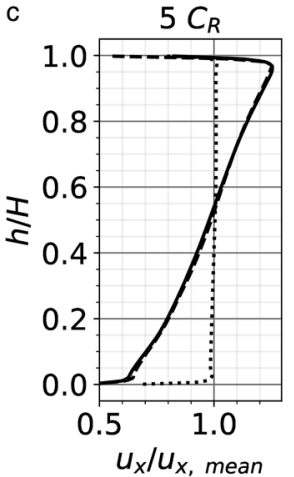

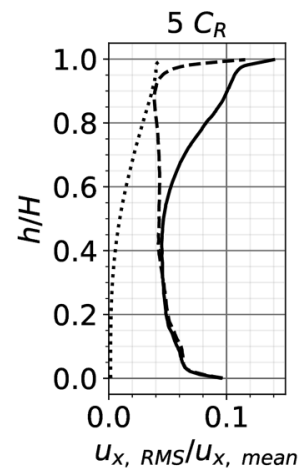

Figure 7. Radial profiles of $u_{x}$ and $u_{x, \text { RMS }}$ on the wake of the fan and OGVs at different axial positions noted in red in Figure 1 . Values are normalised by the average axial velocity $\left(u_{x, \text { mean }}\right)$ at each axial location. $C_{R}$ is the fan blade chord length at 50\% h/H. (solid black) FULLEST; (dashed black) FAN + OGV; (dotted black) CoHP.

which is flat since total variables are imposed at the inlet boundary condition and the inlet of the domain is straight and short to reduce the computational cost. Regarding the RMS values close to the impeller shown in Figure 7f, FAN + OGV matches FULLEST turbulence levels near the hub, whereas they deviate near the shroud due to the rotating shock. The profile for CoHP contains no turbulence near the hub (since no turbulence injection is applied on any simulation) and increases towards the shroud as well because of the shock.

\section{Comparison of the compressor and diffusers predictions}

The interaction from the upstream propagating shock, the fan and OGVs results in an increase $u_{x}$, RMs in the duct (Figure $7 \mathrm{f}$ ) that could potentially distort the shock generation at the leading edge of the impeller. A first attempt to study this interaction is performed by analysing the axial velocity and static pressure spectra at the inlet of the impeller. The spectra depicted in Figure 8 is computed in the static domain and thus it perceives the pressure fluctuations of the shock at the rotating speed of the impeller if this one is considered in the simulation. First, the pressure spectra shows that, FULLEST contains much more broadband background noise with respect to both FAN + OGV and CoHP. This demonstrates once more, that the pressure spectra in the whole forward region of the engine are impacted by the shock either directly by the tones or indirectly by modifying the turbulent structures and possibly increasing its broadband emissions. Secondly, the fan BPF is clearly captured by FULLEST and thirdly, the peaks reach higher amplitudes than for CoHP, especially at higher harmonics. This is possibly an effect of the increased azimuthal content on the 360 degrees FULLEST simulation. Indeed, blade-to-blade shock variations triggered by the turbulence issued from the fan and OGVs could be responsible for significant variations in shock strength that result in higher harmonics similarly to how the buzz-saw noise is generated by blade-to-blade geometric variations (Philpot, 1971; Sofrin and Pickett, 1974; McAlpine et al., 2012). In terms of turbulence, Figure $8 \mathrm{~b}$ shows the spectrum for the axial velocity fluctuations. Since no turbulence injection is applied to the simulations, CoHP shows broadband amplitudes over two orders of magnitude beneath the other simulations. At frequencies below $C_{s}, \mathrm{FAN}+\mathrm{OGV}$ achieves similar values of turbulence than FULLEST despite the above-mentioned differences. 

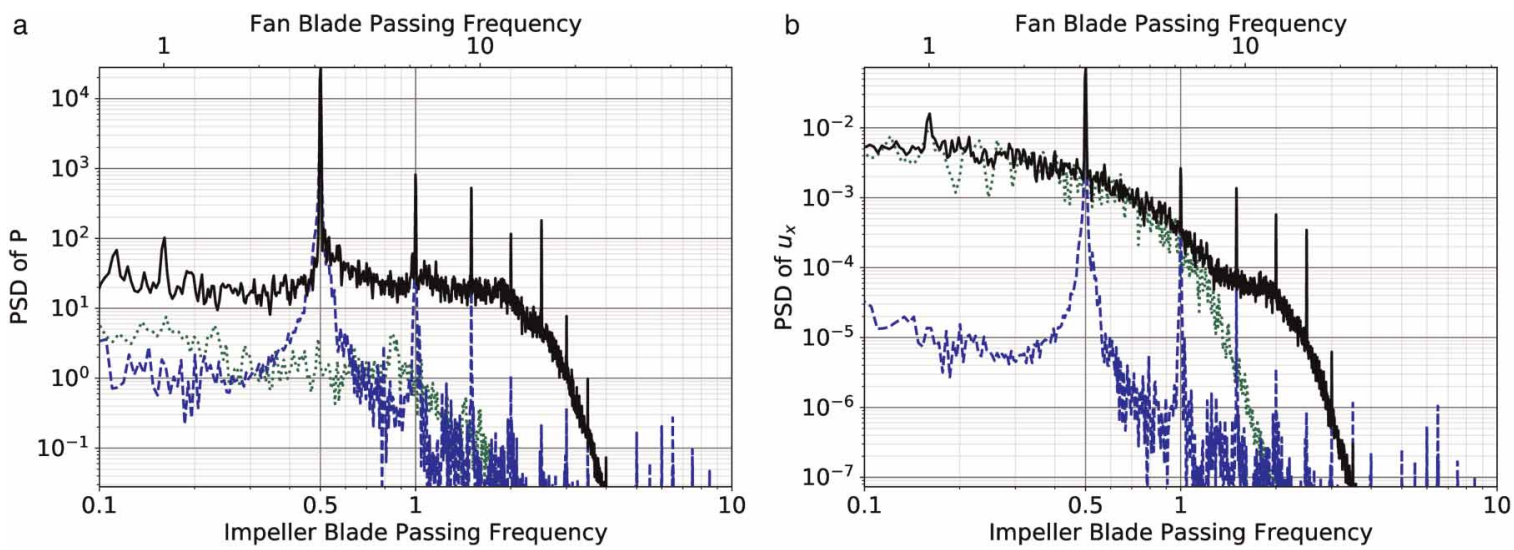

Figure 8. Power spectral density (PSD) at the inlet of the impeller in the static domain as depicted in Figure 1 in green. (solid black) FULLEST, (dotted green) FAN + OGV, (dashed blue) CoHP.

The flow topology is now compared between CoHP and FULLEST. Particular attention is drawn to the topology at the inlet of the impeller and at the interface between the impeller and the radial diffuser. The relative Mach number contours (computed on the relative frame of reference of the impeller) are shown in Figure 9 at different heights of the channel. Overall, results are similar between both simulations. Nevertheless, some specific differences can be identified and these are described in the following for the sake of rigour. At this location, four regions highlighted in Figure 9 can be distinguished. At the leading edge of the main blade the flow accelerates on the suction side and presents a sonic region all along the blade span for FULLEST. In CoHP, this sonic region is less intense and it does not appear at $10 \% \mathrm{~h} / \mathrm{H}$. The flow decelerates and accelerates again in the second region with a broader span over the chord of the blade. This region becomes supersonic at $50 \% \mathrm{~h} / \mathrm{H}$ and merges with the first supersonic bubble at higher $h / H$ values. The flow then encounters the leading edge of the adjacent main blade and is forced to adapt to the pressure at the stagnation point with the shock that propagates upstream. The supersonic region before the shock is more intense and closer to the leading edge of the blade in FULLEST. After passing the leading edge of the main blade, the flow continues to accelerate on the suction side of the blade and reaches the third region where once again the flow is forced to decelerate with a shock between the suction and pressure side of the main blade due to the presence of the leading edge of the splitter blade. This occurs at $90 \% \mathrm{~h} / \mathrm{H}$ with similar patterns between both simulations. Last, on the suction side of the splitter another supersonic bubble appears which merges with the previous region in FULLEST but is
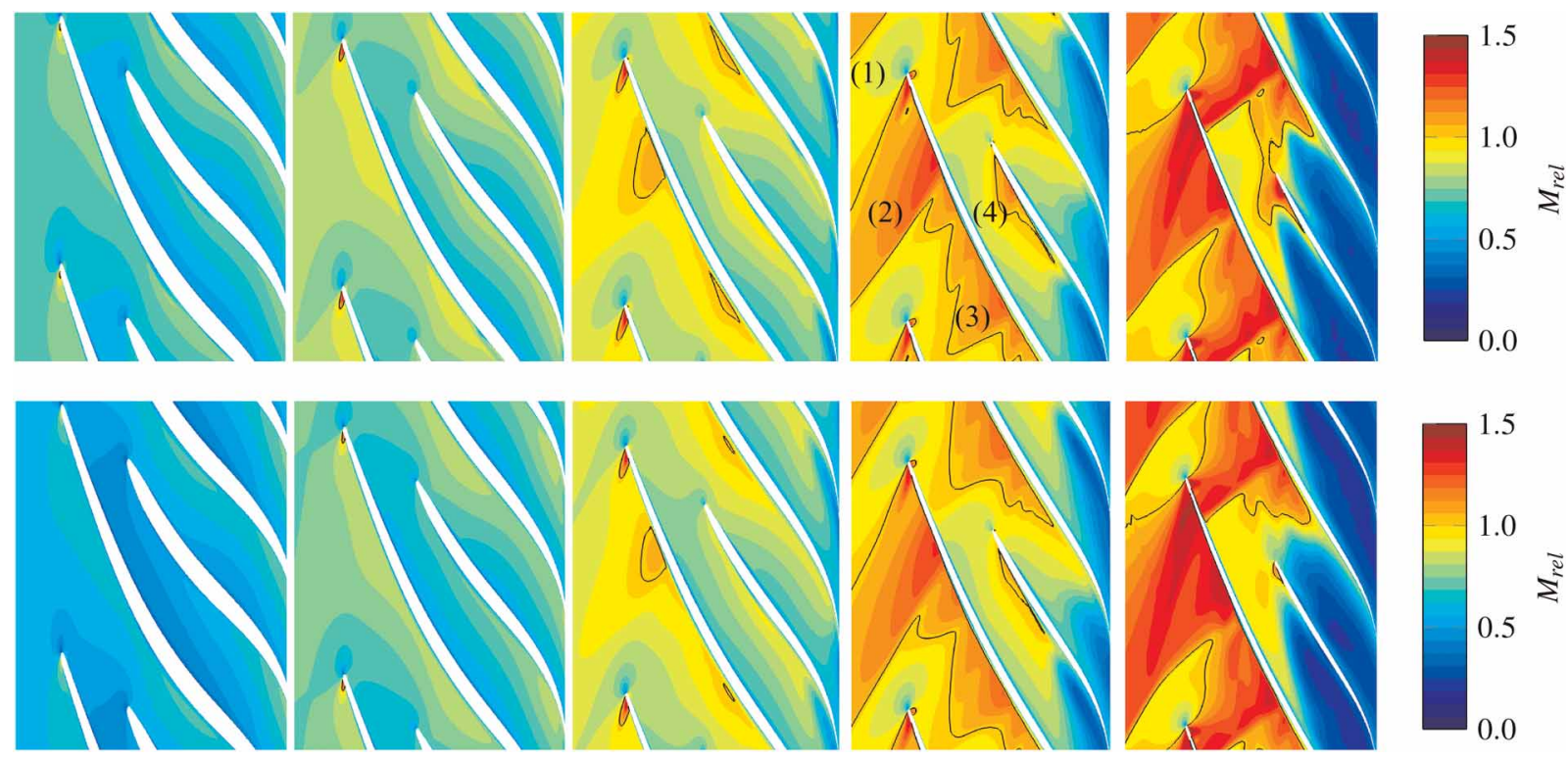

Figure 9. Relative Mach number contours at different $h / H(10 \%, 30 \%, 50 \%, 70 \%$ and $90 \% h / H$ from left to right) for (top) CoHP and (bottom) FULLEST. Black iso-line refers to the sonic line in the relative frame of reference. 
almost not visible in CoHP. The slight changes in flow topology are probably caused by the different velocity profile seen by the impeller as shown in Figure 7c, the differences in background fluctuations shown in Figure 8 and with a lower impact, the differences in the operating point detailed in the companion paper. Since the supersonic bubble is known to be the major source of loss in the impeller, these differences, although small, may have consequences on the performance of the compressor stage.

The waves that propagate through the radial diffuser leave a characteristic pattern in the static pressure RMS for both simulations as depicted in Figure 10 which reaches values up to $50 \mathrm{kPa}$ near the vanes. This level of incoming pressure fluctuations at the combustion chamber inlet is rather large and may have a detrimental effect on combustion instabilities. Although the mean flow has been shown in Figure 9 to be mostly unaffected by the integration of the fan, the pressure RMS is higher in FULLEST. This is expected since the incoming turbulence and pressure fluctuations are higher in FULLEST as illustrated in Figure 8. The flow then goes through the axial diffuser and enters the contouring casing of the combustion chamber.

\section{Comparison of the combustion chamber predictions}

Now that the Fan + OGV and CoHP configurations have been studied, the following section focuses on the combustion chamber. The sectoral stand-alone simulation of the combustion chamber (CC) was performed with a straight inlet. Note that the axial diffuser was not considered since their azimuthal periodicity does not match the one of the combustion chamber and the flow is issued from the radial diffuser with an a priori unknown angle. Furthermore, the CoHP outlet is straight while in reality, the flow issued from the axial diffuser impacts the flame-tube and continues around it. These simplifications have led to some differences between the stand-alone configurations and the integrated simulation FULLEST. Contours of the velocity axial $\left(u_{x}\right)$, radial $\left(u_{r}\right)$ and tangential $\left(u_{\theta}\right)$ cylindrical velocity components at $50 \% \mathrm{~h} / \mathrm{H}$ are compared in Figure 11 for CC, CoHP and FULLEST. Note that in CC and FULLEST the $h / H$ variable smoothly transitions from the height of the axial diffuser channel to the height of the flame-tube casing. In CC, the flow at the inlet is composed only of the axial component. Once it enters the chamber, the $50 \% \mathrm{~h} / \mathrm{H}$ iso-surface crosses a recirculation bubble generated on the top corner of the casing resulting in a negative $u_{x}$. After this point, the flow accelerates vertically as seen by the positive $u_{r}$ to enter the combustor casing space. The flow in the axial diffuser region is similar for both CoHP and FULLEST simulations. Since there are 2 vanes in the radial diffuser (depicted in black) per 5 vanes in the axial diffuser, the flow presents a pattern on $u_{x}$ and $u_{r}$ depending on the direction of the flow issued from the radial diffuser. Three main regions can be distinguished in the axial diffuser vanes shown in Figure 11a. In the first one, the flow issued from the channel R1 is aimed at the channel $\mathrm{C} 1$ reaching a maximum $u_{x}$ in the axial diffuser. The second region shows that the channel R2 is aligned with C3 as well as C4 and the axial velocity is higher on both suction sides of the vanes but lower than in C1. Last, the channels C2 and C5 receive the wake of the radial diffuser vanes and its axial velocity is the lowest at the inlet of the diffuser and separates at mid-chord on the suction side. These patterns are characteristic of the number of vanes in the diffusers which differs from the actual geometry of the real engine. The number of radial and axial vanes were adapted from 19 to 22 and 57 to 55, respectively, to share the same azimuthal periodicity with the impeller. Nonetheless, the comparison between stand-alone and integrated simulations is still valid as both simulations have the same number of vanes. Once the flow passes the axial diffuser, it enters the contouring casing of the combustion chamber. The flow reduces $u_{x}$ as in CC while keeping a trace of the patterns generated by the interaction between the radial and axial diffusers.
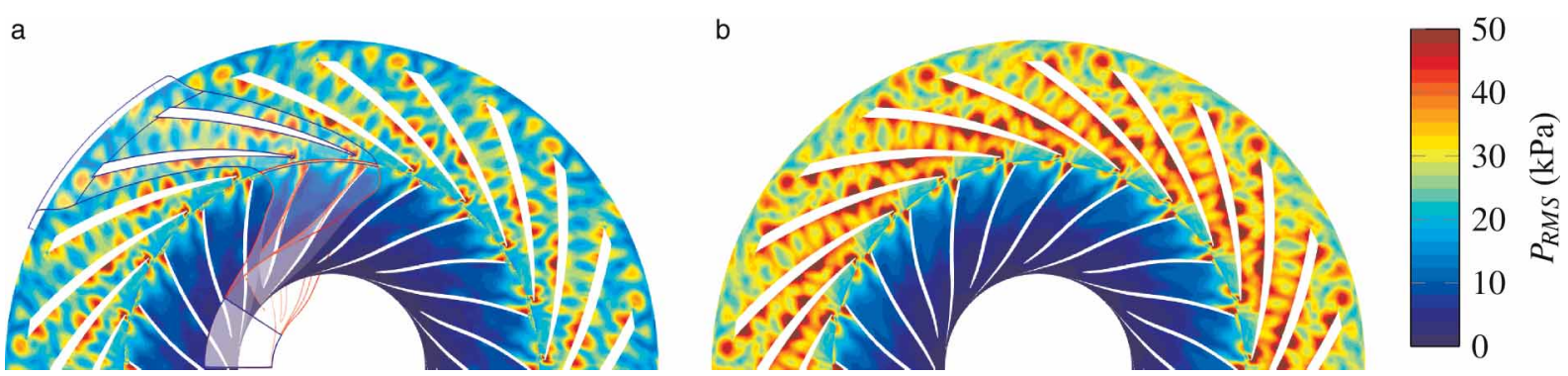

Figure 10. $P_{\mathrm{RMS}}$ contours at $50 \% \mathrm{~h} / \mathrm{H}$ computed on the respective frame of reference of each sub-domain. (a) CoHP. (b) FULLEST. 
$\mathrm{CC}$
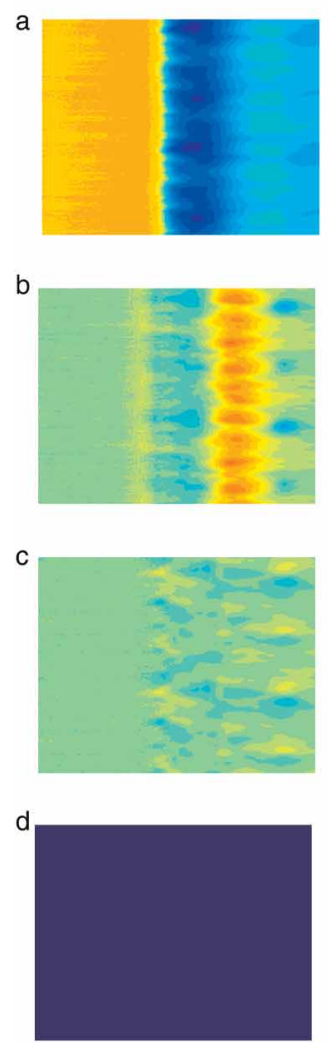

CoHP
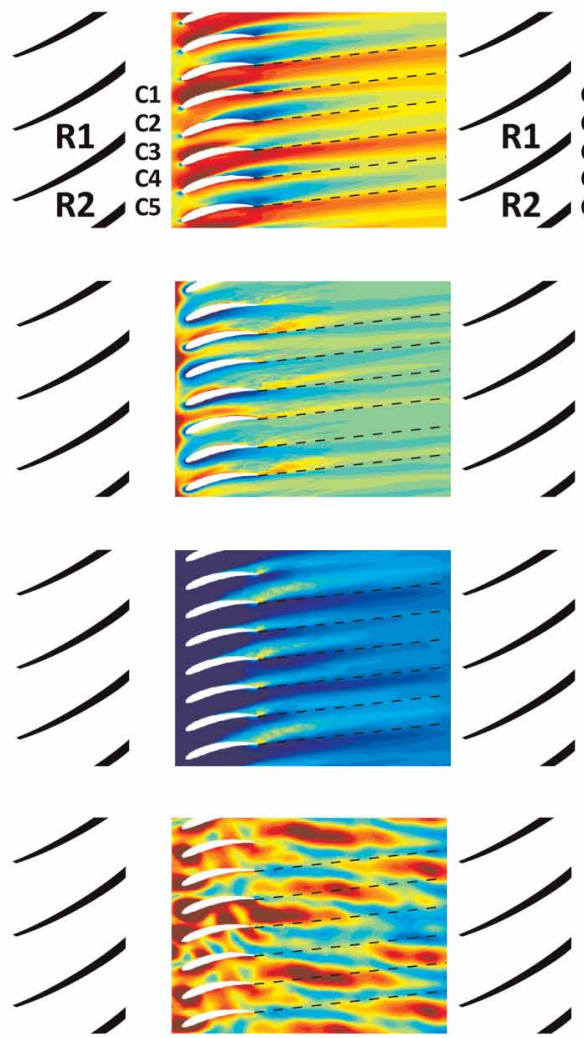

FULLEST
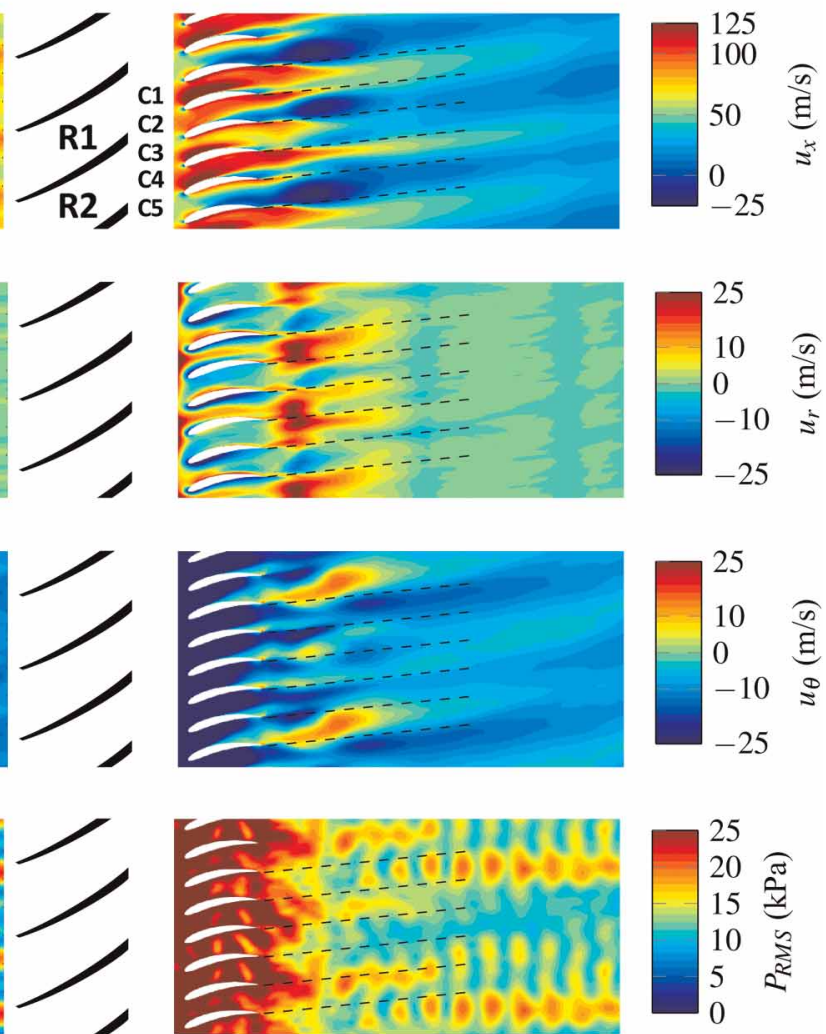

Figure 11. Velocity and $P_{\mathrm{RMS}}$ contours at $50 \% \mathrm{~h} / \mathrm{H}$ at the outlet of the axial diffuser and in the contouring casing of the combustion chamber (if existent) for (left) CC, (centre) CoHP and (right) FULLEST. (dashed black) depicts the flow direction for each vane passage.

The patterns of negative and positive velocities are caused by a combination of the wakes of the radial diffuser with the recirculation bubble and the flame-tube. The above-mentioned recirculation bubble covers the full azimuthal range in CC. In FULLEST, this recirculation region is cut in three by the flow aligned with the radial diffuser vanes (channels $\mathrm{C} 1, \mathrm{C} 2$ and $\mathrm{C} 5$ ) creating 2 separated recirculation bubbles that now interact with the flow below the axial diffuser. The main objective of the axial diffuser is to realign the flow issued from the radial diffuser with the axis of the machine (i.e. reduce $u_{\theta}$ ). However, since the vanes from the axial diffuser are fixed and designed to achieve this at cruise conditions, at take-off conditions, $u_{\theta}$ remains non-zero for both CoHP and FULLEST, which translates to an average residual flow angle of 11 degrees. In CC, $u_{\theta}$ remains around 0 on the full $h / H$ iso-surface, because there is no diffuser and the flow is injected perpendicular to the inlet boundary condition.

The flow interaction between the diffusers illustrated in Figure 11 with the velocity components also impacts the pressure fluctuations generated by the interaction between the impeller and the radial diffuser. Contours of $P_{\text {RMS }}$ are shown in Figure $11 \mathrm{~d}$ for all three simulations. As expected, no fluctuation is detected in CC since the simulations are run without synthetic turbulence injection at the inlets to have a base comparison. In CoHP, the pressure RMS presents an azimuthal and an axial pattern where the flow issued from channel C2 is more intense close to the vanes due to the separation in these vanes. Moving downstream of the vanes, it is the flow issued from channels $\mathrm{C} 1$ and $\mathrm{C} 4$ which presents higher values of RMS. Farther from the vanes, the pattern then shifts azimuthally but because the flow exits with an angle, it is still the flow from channels C1 and C4 as depicted by the dashed lines. In FULLEST, the intensity of the pressure fluctuations is higher on the axial diffuser as it is in the radial diffuser (see Figure 10). The pattern observed in CoHP is altered in FULLEST by an additional axial pattern possibly generated by the interaction of the flow with the wall of the flame-tube. Overall, both CoHP and FULLEST show that realistic boundary conditions should be considered to setup stand-alone simulations of combustion chambers.

An axial cut at plane 31 (plane downstream of the axial diffuser) is shown in Figure 12 to illustrate the velocity components on the full height of the channel. As presented in Figure 11, the uniform velocity components 

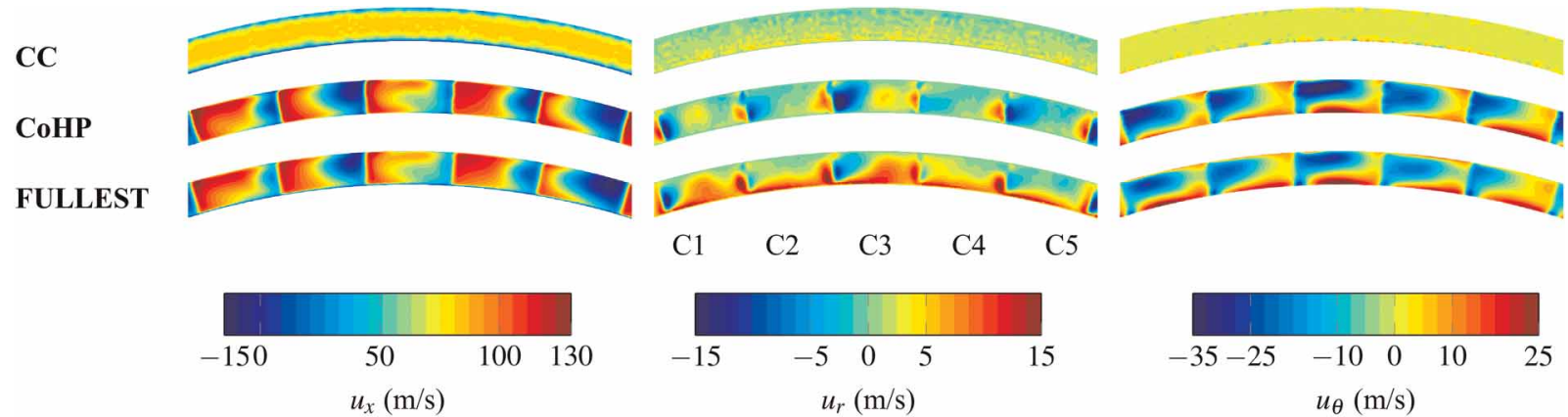

Figure 12. Time-averaged velocity contours (cylindrical components) at plane 31 (trailing-edge of the axial diffuser) noted in blue in Figure 1 for (top) CC, (centre) CoHP and (bottom) FULLEST.

in CC are not representative of the real conditions with the diffusers. Between CoHP and FULLEST, only $u_{r}$ increases near the hub in FULLEST due to the interactions of the flow with the recirculation bubble and the change in direction needed to enter the contouring casing (which is at a greater radius, hence the positive $u_{r}$ ). Similarly, the RMS values of the velocity components depicted in Figure 13 lead to similar conclusions. Since no turbulence has been injected at the inlet of CC, the RMS values are 0 for the stand-alone simulation which are far from the other simulations.

The flow then enters the flame-tube through the top and bottom dilution and primary holes; the swirlers farther back; and through the modelled multi-perforations of the flame-tube (Bizzari et al., 2018). In this case, each modelled perforation is set to either a constant suction or an injection of mass-flow rate at a determined temperature by the flame-tube side. The actual value of each perforation flow-rate is computed in a preprocessing step and does not change during the simulation. This means that with the actual modelling, and possibly contrary to real engines, the pressure fluctuations issued from the compressor cannot modify the mass-flow of each perforation. This is not the case for the dilution and primary holes or the swirler, which are meshed. The PSD of the mass-flow passing through the top primary hole and the swirler is shown in Figure 14. At low frequencies, both simulations achieve similar amplitudes. In FULLEST, the mass-flow entering the flame-tube fluctuates at the impeller BPF as well as harmonics and overall presents higher levels at high frequencies. This means that the pressure fluctuations incoming from upstream of the combustion chamber have an effect on the mass flow distribution of the dilution holes, and hence within the flame tube.

Inside the flame-tube, the time-averaged fields are barely impacted by the integration of the other components. This is illustrated by comparing the time-averaged static pressure, temperature and their RMS values for a meridional cut traversing the swirler in Figure 15. As expected, the most significant change comes from the pressure RMS as depicted in Figure 15b. As shown above, the pressure fluctuations propagate through the contouring casing of the combustion chamber. The same behaviour was reported by Pérez Arroyo et al. (2020) in an annular combustion chamber with a T-shaped vaporizer instead of a swirler. In FULLEST, the pressure fluctuations enter the flame-tube as can be seen by the higher levels of $P_{\mathrm{RMS}}$ around the flame-tube wall. Slight differences are observed for the other variables which are mostly due to a change in angle of the jets issued from the primary and dilution holes as explained in the following.

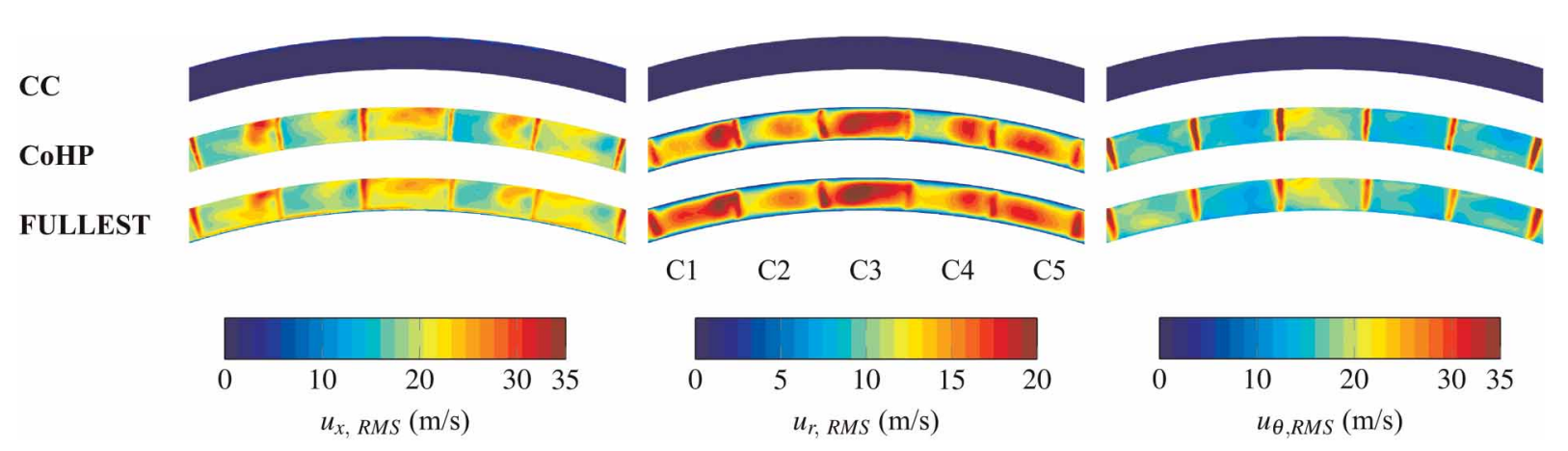

Figure 13. RMS velocity contours (cylindrical components) at plane 31 (trailing-edge of the axial diffuser) noted in blue in Figure 1 for (top) CC, (centre) CoHP and (bottom) FULLEST. 

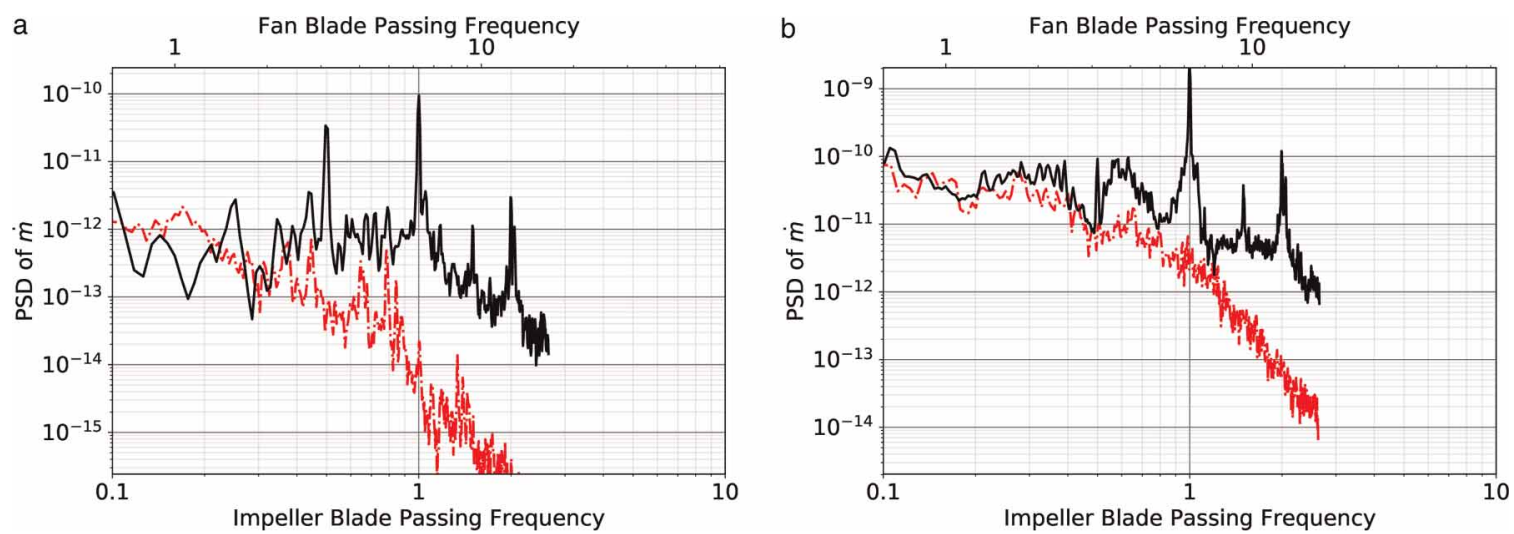

Figure 14. Power spectral density (PSD) of the integral mass-flow rate $\dot{m}$ on different planes. (solid black) FULLEST, (dashed red) CC. (a) On the external primary hole. (b) At the exit of the swirler.

As shown in Figure 11, the angle at which the flow enters the contouring casing is different depending on whether or not the domain contains the axial diffuser. This change in $u_{\theta}$ impacts the ejection angle of the dilution and primary holes as illustrated in Figure 16 by time-averaged and RMS Mach number $\left(M_{\mathrm{RMS}}\right)$ contours in axial cuts passing by both the dilution and primary holes. From these cuts, it can be observed that the angle of ejection in FULLEST changes roughly by 3 degrees with respect to the perpendicular direction defined by the flame-tube wall in CC. In addition, the Mach number is slightly higher and reaches a longer extent inside the flame-tube in FULLEST. Similarly, the RMS values increase in FULLEST mainly at the primary hole cut. These minor differences can be seen in the contours from Figure 15. For example, $T_{\text {RMS }}$ in FULLEST at the exit of the top dilution hole is less intense because the jet moves away from this plane.

Pressure fluctuations entering the flame-tube can be measured in the spectra at all locations. Figure 17 shows the spectra at four locations depicted in Figure 1 which correspond to a location where the gases are hot (Figure 17a), cold (Figure 17b) and at the exit of the combustion chamber (Figure 17c and 17d). The spectral content in the hot region has been substantially modified with respect to CC. In the stand-alone simulation, two broadband peaks arise at low and high frequencies. In FULLEST, the background amplitude is already higher than these peaks, specially at high frequencies. In addition to the peaks at the impeller BPF and harmonics, additional peaks are found at lower frequencies. Further analyses are needed to ascertain the origin of these supplementary peaks, which will be performed in the future with a longer simulated physical time to help for a better convergence of the low frequency range. In the cold region (Figure 17b), these peaks are masked by the background levels which are one order of magnitude above the levels in the hot region. The impeller BPF and harmonics are still visible in FULLEST and CC spectrum no longer presents broadband peaks and instead follows closely the spectrum in FULLEST. At the exit of the combustion chamber, some of the additional peaks are again visible in FULLEST since the background fluctuations have decayed. At this location, the impeller BPF peak is still about 4 orders of magnitude greater than the background. These fluctuations will be transported
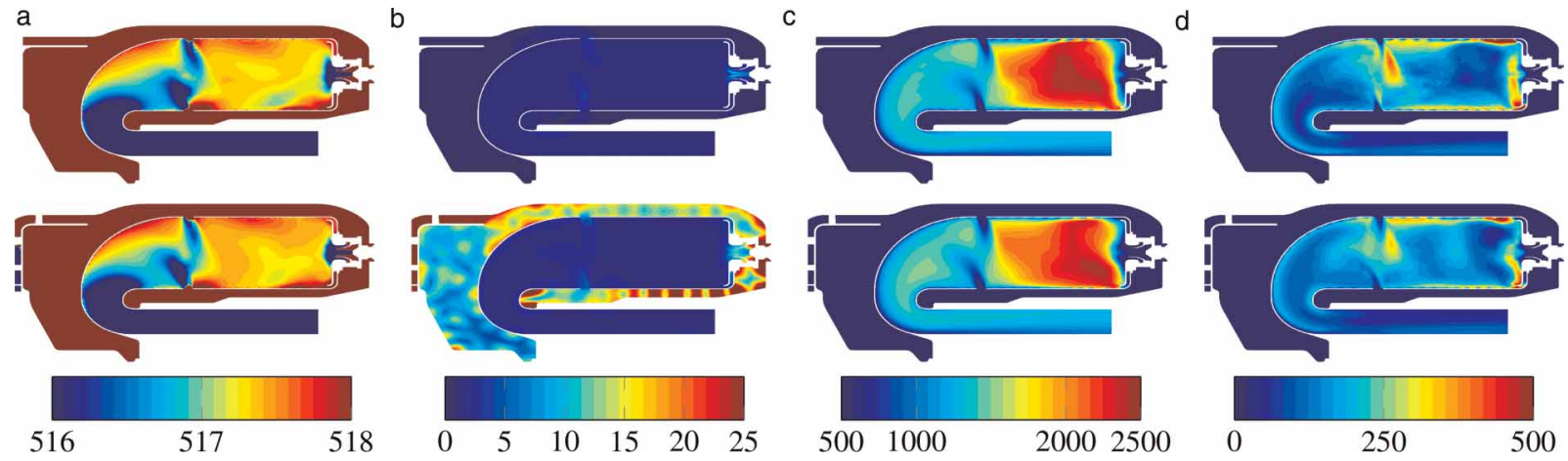

Figure 15. Mean and RMS contours at a meridional plane located at the centre of the swirler (top) CC and (bottom) FULLEST. (a) $P$ (kPa). (b) $P_{\text {RMS }}(\mathrm{kPa})$. (c) $T$ (K). (d) $T_{\text {RMS }}(\mathrm{K})$. 
CC

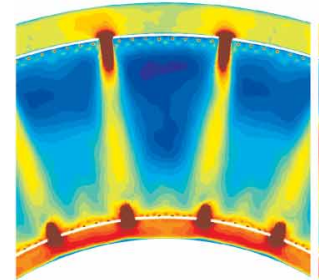

FULLEST

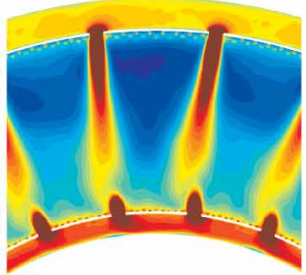

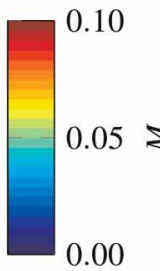

$\mathrm{CC}$

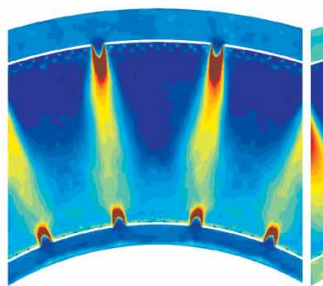

FULLEST

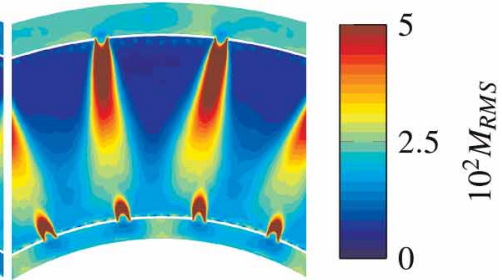

Figure 16. Mean and RMS Mach number contours at axial planes (perpendicular to the axis of the machine) located at the primary holes. The observer is looking from the location of the fan.

through the turbine stage and eventually radiate to the far-field. This peak and the first harmonic are also visible in the static temperature PSD as shown in Figure 17d, however, its impact on the performance of the turbine stage should be negligible since its amplitude is 2 orders of magnitude lower than the values obtained at the low frequency range. In CC, both pressure and temperature PSD present similar trends but without the tonal content.

The exit of the combustion chamber (noted as plane 40) is further analysed in the following. This is a location of interest because it is also the inlet of the turbine stage (in the real engine). The information from this plane is commonly exchanged between design teams from the combustion and turbine departments and it is of crucial importance for the design of the turbine because non-uniform turbulence levels, residual swirl as well as temperature hot-spots may significantly impact the turbine life cycle (Dorney et al., 1999; Povey and Qureshi, 2009; Ireland and Dailey, 2010). Figure 18 depicts the contours at plane 40 for the time-averaged and RMS total pressure and temperature for both simulations. No difference is found for the time-averaged total pressure $P_{t}$ (Figure 18a). On the other hand, the RMS values (Figure 18c) are shifted by about 14 degrees for FULLEST if compared to the CC position. This angle is half the periodicity and is a direct result of the pressure fluctuations that enter the flame-tube through the dilution holes which are separated about 14 degrees apart. The time-
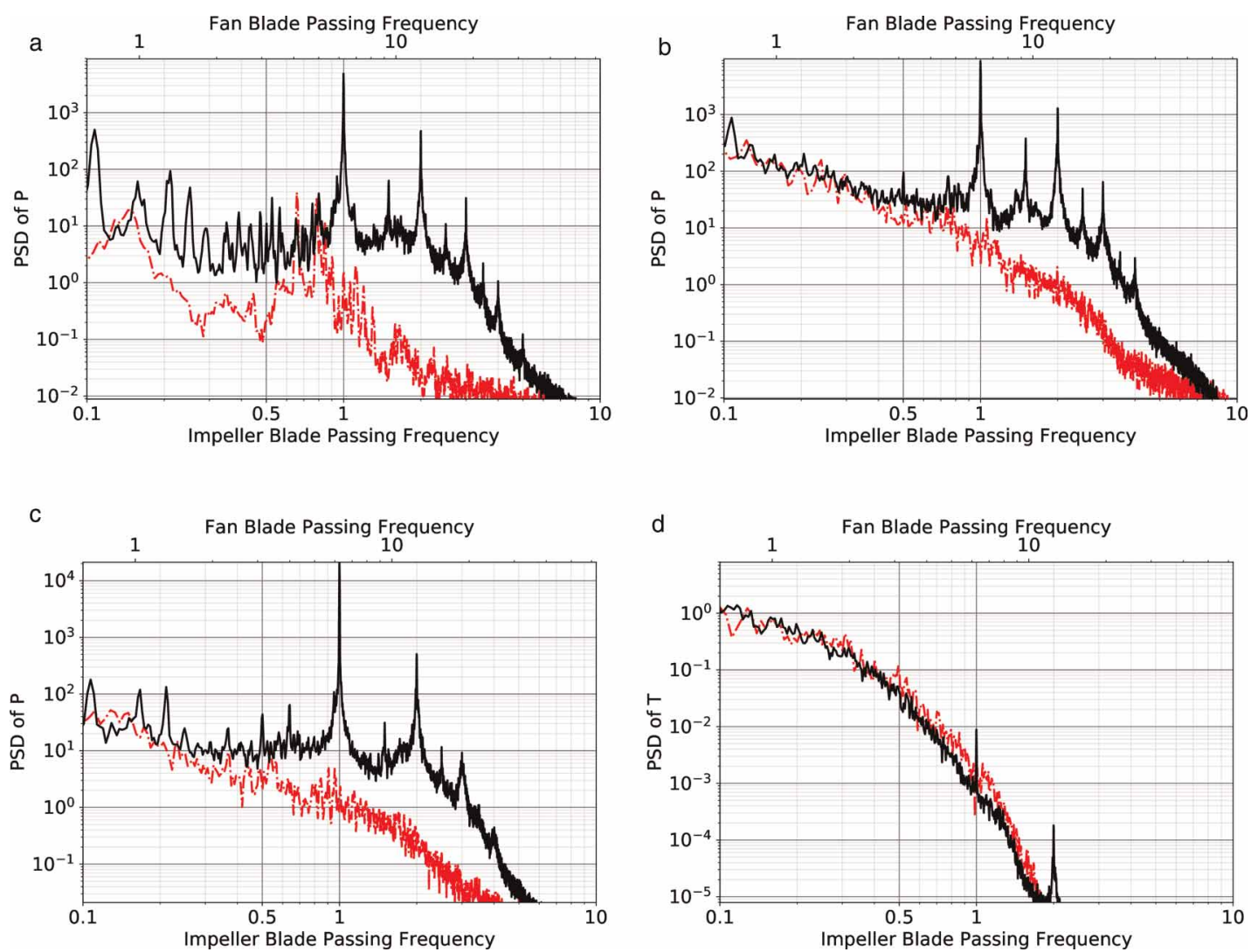

Figure 17. Power spectral density (PSD) at different locations noted in purple in Figure 1. (solid black) FULLEST, (dashed red) CC. (a) Hot region. (b) Cold region. (c) Exit of the flame-tube. (d) Exit of the flame-tube. 

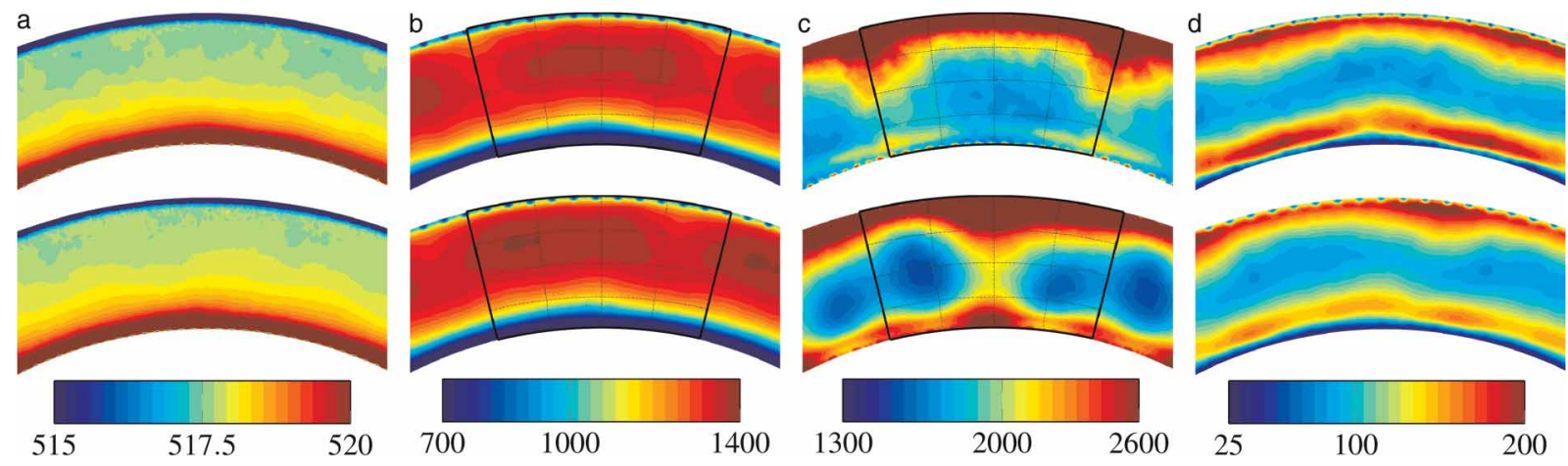

Figure 18. Mean and RMS total pressure and temperature contours at axial planes (perpendicular to the axis of the machine) located at plane 40 noted in orange in Figure 1 for (top) CC and (bottom) FULLEST. The observer is looking from the location of the fan. Black lines depict 1 sector of the combustion chamber centred on the plane of the swirler. (a) $P_{t}$ (kPa). (b) $T_{t}(\mathrm{~K})$. (c) $P_{t, \mathrm{RMS}}(\mathrm{Pa})$. (d) $T_{t, \mathrm{RMS}}(\mathrm{K})$.

averaged total temperature $T_{t}$ illustrated in Figure $18 \mathrm{~b}$ presents as well an azimuthal shift but of only 7 degrees. In this case, this shift is caused by the change in angle of the jets issued from the primary and dilution holes depicted in Figure 16. Values of $T_{t, \text { RMS }}$ are characterised in both cases by higher levels near the hub and the casing which are generated by the mixing of the cold jets from the multi-perforated flame-tube (with the thickened-hole model by Bizzari et al. (2018)) and the hot flow from combustion. These jets can be seen in the shroud of Figure $18 \mathrm{~b}$ and $18 \mathrm{~d}$.

\section{Conclusions}

The effects of the integration of several engine components is investigated with an integrated reactive large-eddy simulation with over 2,100 million cells of a 360 azimuthal degrees gas-turbine including the fan, radial compressor and annular chamber. This is performed by comparing the results against their stand-alone counterparts.

Even though there is no impact of the integration on the operating point of the machine (Pérez Arroyo et al., 2021), the average, RMS and spectra are greatly impacted by this process. This is clearly demonstrated by the rotating shock generated on the impeller leading edge at take-off conditions. The shock propagates upstream and interacts with the fan and OGVs. Stand-alone simulations of the fan component are not able to capture this phenomenon by construction unless better physical outlet boundary conditions are implemented. Note that the interaction between the high-pressure compressor and the fan can be deduced from the experimental spectra by Brown and Sutliff (2018). The shock is not only seen in the spectra but also affects the shape of the mean flow and RMS values. Indeed, turbulence generation on the fan blades and on the bypass duct, is modified as shown by the change in the position of the vortical structures in the fan wake, which some of them are excited at the frequency of the rotating shock. The authors recognise that the impact of the integration is enhanced by the fact that the engine is running at off-design conditions, however, it is at these operating points where LES could bring additional value since they are able to capture unsteady phenomena that may not be accounted for in the design process or with lower order models. Furthermore, the integration of the fan with the compressor generates a feedback on the compressor since the shock modifies the turbulence and background pressure fluctuations propagated from the OGVs into the impeller which highlights again the need of more realistic boundary conditions. The compressor itself is barely impacted by the integration and only the background pressure RMS increases in FULLEST. The integration of the combustion chamber deals with geometrical differences between stand-alone and FULLEST domains. Because the axial diffuser is not modelled in CC, the flow enters axially, which is not the case for FULLEST. At design conditions, the axial diffuser aims to realigning the flow with the axis of the machine. This is however not ensured for other operating points as shown in FULLEST. In this case, the difference in angle also affects the ejection angle of the jets generated on the dilution or primary holes and the angular position of the hot-spot that will impact the eventual turbine. The geometrical difference is not only the missing axial diffuser in CC but also the missing radial diffuser. The flow issued from the radial diffuser modifies the flow of the axial vanes and the flow that enters the contouring casing of the combustion chamber. Even though the effect of integration is minimal because the flow in the annular chamber does not directly enter the flametube, it could have an effect if the patterns align with the dilution and primary holes that connect with the flame-tube. Finally, integrating the combustion chamber with the compressor increases the spectral tonal content 
at all locations of the combustion chamber with peaks at the impeller BPFs and harmonics. This impact is though negligible for combustion as already demonstrated by Pérez Arroyo et al. (2020) in another configuration but may have detrimental consequences for the core-noise of the machine if the azimuthal content is modified (Boyle et al., 2020).

Finally, it is concluded that the study of integration effects of several components of an engine with LES is possible. The authors acknowledge that some effects of the integration are specific to this engine and simulation (i.e. the shock generated at the impeller and the lack of axial diffuser vanes in CC). Nonetheless, it gives a general idea of how non-physical boundary conditions often used in stand-alone simulations affect the flow. This work is a first step towards the LES of a full digital twin of the engine where effect of integration of the turbine stages and the exhaust jet are also considered.

\section{Nomenclature}

\section{Symbols}

$C_{R} \quad$ Rotor chord length (m)

$C_{s} \quad$ Peak at half the impeller BPF generated by the upstream propagating shock (-)

$h / H$ Normalised hub-to-shroud distance (-)

$\dot{m} \quad$ Mass-flow $(\mathrm{kg} / \mathrm{s})$

$M \quad$ Mach number (-)

$M_{\text {rel }} \quad$ Relative Mach number (-)

$P \quad$ Static pressure $(\mathrm{Pa})$

$P_{t} \quad$ Total pressure $(\mathrm{Pa})$

$T \quad$ Static temperature $(\mathrm{T})$

$T_{t} \quad$ Total temperature (K)

$\theta / \theta_{s}$ Normalised azimuthal angle with the azimuthal-sector angle $(\mathrm{K})$

$u_{x} \quad$ Axial velocity $(\mathrm{m} / \mathrm{s})$

$u_{r} \quad$ Radial velocity $(\mathrm{m} / \mathrm{s})$

$u_{\theta} \quad$ Tangential velocity $(\mathrm{m} / \mathrm{s})$

\section{Acronyms}

BPF Blade-Passing Frequency

FULLEST First fUlL engine computation with Large Eddy SimulaTion

LES Large-Eddy Simulation

OGV Outlet-guide vane

PSD Power Spectral Density

RMS Root-mean-square

TLV Tip-leakage vortices

\section{Acknowledgments}

The authors would like to thank T. Quirante and N. Vieira-Nobre from Akira Technologies for providing the geometry and operating conditions, and N. Buffaz and A. Paillassa from Safran Helicopter Engines for their technical support on the generation of the reduced-count geometry of the compressor and for performing its respective Reynolds-Averaged Navier-Stokes simulations. The authors are also grateful to G. Exilard and S. Richard from Safran Helicopter Engines for his help during the setup of the perforations in the combustion chamber.

\section{Funding sources}

These results benefited from funding or developments from projects ATOM (DGAC/SafranTech No. 2018-39), EXCELLERAT (H2020 No. 823691) and EPEEC (H2020 No. 801051). This work was granted access to the HPC resources of GENCI/TGCC (Joliot-Curie supercomputer) on the PRACE allocation project FULLEST (Project No. RA5191). 


\section{Competing interests}

Carlos Pérez Arroyo declares that he has no conflict of interest. Jérôme Dombard declares that he has no conflict of interest. Florent Duchaine declares that he has no conflict of interest. Laurent Gicquel declares that he has no conflict of interest. Benjamin Martin declares that he has no conflict of interest. Nicolas Odier declares that he has no conflict of interest. Gabriel Staffelbach declares that he has no conflict of interest.

\section{References}

Adamczyk J. J. (1984). Model equation for simulating flows in multistage turbomachinery, NASA, Technical Memorandum.

Bizzari R., Lahbib D., Dauptain A., Duchaine F., Gicquel L., and Nicoud F. (2018). A thickened-hole model for large eddy simulations over multiperforated liners. Flow, Turbulence and Combustion. 101 (3): 705-717. https://doi.org/10.1007/s10494-018-9909-3.

Boyle D. K., Henderson B. S., and Hultgren L. S. (2020). DGEN aeropropulsion research turbofan core/combustor-noise measurements: Experiment and modal structure at core-nozzle exit. In: Turbomachinery Technical Conference and Exposition, American Society of Mechanical Engineers.

Brown C. A. and Sutliff D. L. (2018). DGEN aeropropulsion research turbofan (DART): Lossless projection of measured engine noise spectra to a 1-foot-radius arc. In: 2018 AIAA/CEAS Aeroacoustics Conference, p. 3280.

Dapogny C., Dobrzynski C., and Frey P. (2014). Three-dimensional adaptive domain remeshing, implicit domain meshing, and applications to free and moving boundary problems. Journal of Computational Physics. 262: 358-378. https://doi.org/10.1016/j.jcp.2014.01. 005 .

Daviller G., Brebion M., Xavier P., Staffelbach G., Müller J.-D., and Poinsot T. (2017). A mesh adaptation strategy to predict pressure losses in les of swirled flows. Flow, Turbulence and Combustion. 99 (1): 93-118. https://doi.org/10.1007/s10494-017-9808-z.

Dorney D. J., Gundy-Burlet K. L., and Sondak D. L. (1999). A survey of hot streak experiments and simulations. International Journal of Turbo and Jet Engines. 16 (1): 1-16. https://doi.org/10.1515/TJJ.1999.16.1.1.

Inoue M. and Kuroumaru M. (1989). Structure of tip clearance flow in an isolated axial compressor rotor. Journal of Turbomachinery. 111: 250-256. https://doi.org/10.1115/1.3262263.

Ireland P. and Dailey G. (2010). Aerothermal performance of internal cooling systems in turbomachines: Von Karman Institute Lecture Series, Internal cooling in turbomachinery. Rhode-Saint-Genèse, Belgium.

McAlpine A., Schwaller P., Fisher M., and Tester B. (2012). "buzz-saw" noise: Prediction of the rotor-alone pressure field. Journal of Sound and Vibration. 331 (22): 4901-4918. https://doi.org/10.1016/j.jsv.2012.06.009.

Müller J.-D. (1999). Coarsening 3-D hybrid meshes for multigrid methods. In: 9th Copper Mountain Multigrid Conference, Citeseer.

Papadogiannis D., Duchaine F., Sicot F., Gicquel L., Wang G., and Moreau S. (2014). Large eddy simulation of a high pressure turbine stage: Effects of sub-grid scale modeling and mesh resolution. In: Turbo Expo: Power for Land, Sea, and Air, Vol. 45615, American Society of Mechanical Engineers, p. V02BT39A018.

Pérez Arroyo C., Dombard J., Duchaine F., Gicquel L., Odier N., et al. (2020). Large-eddy simulation of an integrated high-pressure compressor and combustion chamber of a typical turbine engine architecture. In: Turbomachinery Technical Conference and Exposition, American Society of Mechanical Engineers.

Pérez Arroyo C., Dombard J., Duchaine F., Gicquel L., Martin B., et al. (2021). Towards the large-eddy simulation of a full engine: Integration of a 360 azimuthal degrees fan, compressor and combustion chamber. Part I: Methodology and initialisation. Global Power and Propulsion Society. 5: 1-16.

Philpot M. (1971). The buzz-saw noise generated by a high duty transonic compressor.

Povey T. and Qureshi I. (2009). Developments in hot-streak simulators for turbine testing. Journal of Turbomachinery. 131 (3): 031009. https://doi.org/10.1115/1.2987240.

Schönfeld T. and Rudgyard M. (1999). Steady and unsteady flow simulations using the hybrid flow solver AVBP. AIAA Journal. 37 (11): 1378-1385. https://doi.org/10.2514/2.636.

Sharma O., Butler T., Joslyn H., and Dring R. (1985). Three-dimensional unsteady flow in an axial flow turbine. Journal of Propulsion and Power. 1 (1): 29-38.

Sofrin T. and Pickett G. (1974). Multiple pure tone noise generated by fans at supersonic tip speeds, NASA, Technical Memorandum. 